\title{
Statistical Analysis of Excitonic Transitions in Single, Free- Standing GaN Nanowires: Probing Impurity Incorporation in the Poissonian Limit
}

\author{
Carsten Pfüller ( $\varangle$ ), Oliver Brandt, Timur Flissikowski, Caroline Chèze, Lutz Geelhaar, Holger T. Grahn, and \\ Henning Riechert
}

Paul-Drude-Institut für Festkörperelektronik, 5-7 Hausvogteiplatz, Berlin 10117, Germany

Received: 3 August 2010 / Revised: 13 October 2010 / Accepted: 17 October 2010

C The Author(s) 2010. This article is published with open access at Springerlink.com

\begin{abstract}
Single, free-standing GaN nanowires grown by plasma-assisted molecular-beam epitaxy have been investigated with low temperature micro-photoluminescence. The quantitative analysis of the luminescence spectra of around 100 nanowires revealed that each nanowire exhibits its own individual spectrum. A significant fraction of nanowires exclusively emits at energies corresponding to either surface-donor-bound or free excitons, demonstrating that optical properties of individual nanowires are determined by a few impurity atoms alone. The number of impurities per nanowire and their location within the nanowires varies according to Poissonian statistics.
\end{abstract}

\section{KEYWORDS}

Single nanowires, impurities, doping, excitonic luminescence, scaling

\section{Introduction}

Since the advent of microelectronics, the increase in package density and performance of integrated circuits has been driven by the continuous downsizing of $\mathrm{Si}$ technology as specified by the International Technology Roadmap for Semiconductors (ITRS) [1]. According to the ITRS, a physical gate length of classical complementary metal-oxide-semiconductor (CMOS) structures of less than $10 \mathrm{~nm}$ is expected for the year 2016. Simultaneously, alternative device concepts ("More-than-Moore") such as carbon nanotubes (CNTs) or semiconductor nanowires (NWs) are attracting great interest. The latter show particular promise due to their compatibility with standard Si technology [2], and the possibility of defining three-dimensional device architectures by combining axial and radial heterostructures. Various devices, such as light emitting diodes, lasers, field effect transistors, photodetectors [3], and solar cells [4] have already been demonstrated using both as-grown NW ensembles and (dispersed) single NWs.

Independent of the nature of the above mentioned devices (i.e., top-down or bottom-up), their functionality depends on the rigorous control of impurity incorporation. Intentional doping to a certain, welladjusted level defines the semiconducting properties of the material, whereas unintentional impurities may

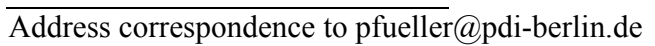


act as traps or nonradiative recombination centers with detrimental consequences for the performance of the device. The doping densities involved are typically at the ppm level, while the concentration of undesired elements should not exceed the ppb range. The implications for nanometer-scaled device components (such as NW-based ones) are obvious: the ongoing miniaturization will inevitably result in devices with a single-digit number of impurity atoms per component. The statistical nature of impurity incorporation thus gives rise to concerns about the resulting variations of the optical and electrical properties between devices. In the extreme case of the total absence of impurity atoms, the properties of the material are so drastically different from the doped one that the device may not function as intended.

Here, we analyze the spontaneous emission of a large number of single, free-standing GaN NWs with the goal of monitoring the inevitable statistical fluctuations of impurity incorporation into these nanoobjects.

\section{Experimental}

The NWs were grown self-induced (catalyst-free) by molecular beam epitaxy (MBE) on two differently oriented Si substrates [sample A on $\mathrm{Si}(001)$ and sample B on $\mathrm{Si}(111)$ ] [5]. Further details are given in the Methods section below. The investigated NWs covered a mesoscopic area $(200 \mu \mathrm{m} \times 200 \mu \mathrm{m})$ of these substrates, and temperature gradients or flux variations across the investigated area are hence vanishingly small. In other words, the NWs are fabricated under conditions of maximum cleanliness and exceptionally high uniformity. Based on our previous work [6], we estimate the absolute upper limit for the concentration of unintentional donors to be less than $5 \times 10^{16} \mathrm{~cm}^{-3}$. For this concentration, for a diameter of about $30 \mathrm{~nm}$, and for a maximum NW length of $300 \mathrm{~nm}$, less than five donors per NW are expected on average. Such low impurity concentrations can be detected only by optical means. As a matter of fact, the presence and number of donors determine the spectral and temporal characteristics of excitonic recombination. Therefore, photoluminescence (PL) experiments are ideally suited to detect impurity fluctuations from NW to NW. Due to the low NW density of less than $1 \mu \mathrm{m}^{-2}$, we are able to optically address single NWs. In order to investigate a larger number of free-standing NWs, we performed micro-photoluminescence ( $\mu$-PL) area maps across 100 NWs. Further details are given in the Methods section below.

\section{Results and discussion}

Exemplary PL spectra of individual NWs of samples A and B are shown in Figs. 1(a) and 1(b). We can assign the observed emission lines to definite decay channels because the NWs are free of strain. The absence of strain follows from the principle of Saint-Venant [7] which can be stated as follows: if biaxial stress is applied to one end of an otherwise free elastic rod of diameter $d$ this stress will decay to zero approximately within a distance $d$ from the clamped end of the rod.

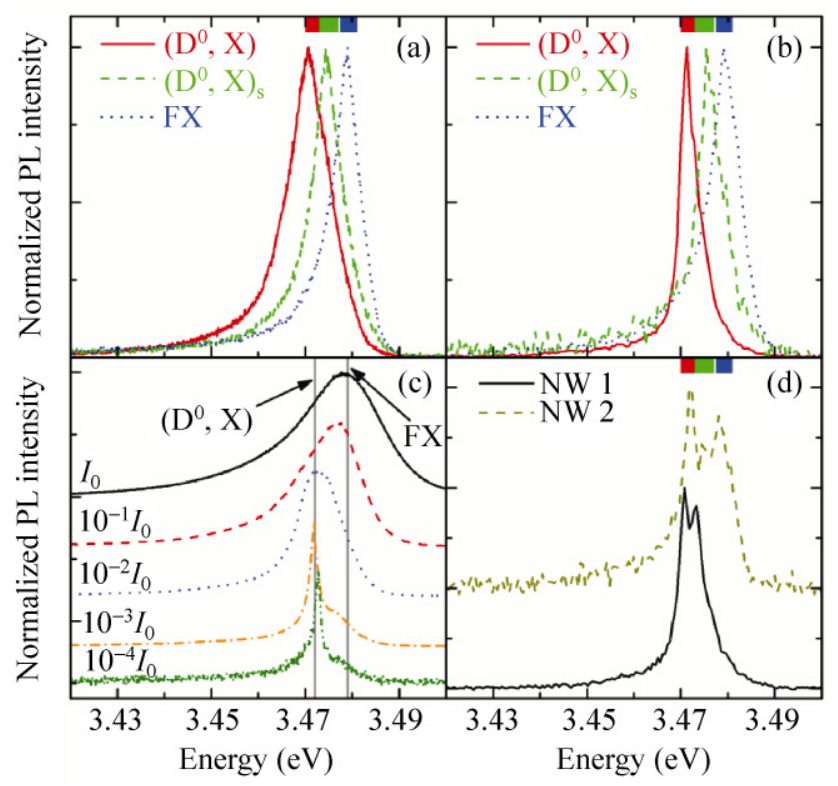

Figure 1 Typical spectra of free-standing NWs, which are dominated by $\left(\mathrm{D}^{0}, \mathrm{X}\right)$ (red), $\left(\mathrm{D}^{0}, \mathrm{X}\right)_{\mathrm{s}}$ (green), and $\mathrm{FX}$ (blue) luminescence, from (a) sample A grown on $\mathrm{Si}(001)$ and (b) sample $\mathrm{B}$ grown on $\mathrm{Si}(111)$. The spectra have been extracted from the respective area maps in Fig. 2(a). (c) PL spectra of a single freestanding GaN NW from sample A at various excitation densities as indicated in the figure. (d) PL spectra of two single free-standing NWs from sample $B$, which exhibit multiple excitonic transitions. The $\left(\mathrm{D}^{0}, \mathrm{X}\right),\left(\mathrm{D}^{0}, \mathrm{X}\right)_{\mathrm{s}}$, and $\mathrm{FX}$ energy ranges are marked by the red, green, and blue bars, respectively 
In other words, only the very bottom of the NW close to the interface with the substrate may experience strain. However, cathodoluminescence measurements (not shown here) reveal that the bottom $50-100 \mathrm{~nm}$ of the NW do not emit any luminescence at all, possibly due to an escape of photogenerated carriers into the $\mathrm{Si}$ substrate or due to the presence of misfit dislocations/ dangling bonds at the NW/substrate interface. In any case, the measured luminescence originates exclusively from the unstrained part of the NW.

Three different energy ranges corresponding to three distinct decay channels are distinguished. First, the recombination of free excitons $(\mathrm{FX})$ in unstrained GaN occurs at $3.479 \mathrm{eV}$ [8]. Second, when free excitons are bound to donors, their transition energy is reduced by their binding energy to the donor. In the case of excitons bound to bulk-like shallow donors (i.e., with a distance from the surface that is larger than the exciton Bohr radius $a_{\mathrm{B}}$ ), this binding energy amounts, depending on chemical nature of the donor, to $6-8 \mathrm{meV}$. The decay of the excitonic complex $\left(\mathrm{D}^{0}, \mathrm{X}\right)$ thus results in an emission line located between 3.471 and $3.473 \mathrm{eV}$ [8]. Note that dielectric confinement as studied by, for example, Muljarov et al. [9] does not play any role for this bulk-like $\left(\mathrm{D}^{0}, \mathrm{X}\right)$ in these $\mathrm{NWs}$, as their diameter still vastly exceeds the exciton Bohr radius. Third, when a donor resides close to the surface, the binding energy of the associated excitonic complex $\left(\mathrm{D}^{0}, \mathrm{X}\right)_{\mathrm{s}}$ is reduced $[10,11]$ and its transition energy is increased, as discussed in detail in Ref. [6]. The reduction of binding energy persists also in the presence of a dielectric mismatch, which now becomes important, independent of the actual dimensions of the NW [11]. The actual transition energy of the $\left(\mathrm{D}^{0}, \mathrm{X}\right)_{\mathrm{s}}$ complex depends monotonically on the distance of the related donor from the NW sidewall and is here assumed to span the whole energy range from the bulk-like donor-bound to the free exciton (3.473-3.477 eV). This assumption is reasonable, since the surface-donor binding energy may be reduced to $25 \%$ of the bulk value [10], which translates to a maximum blueshift of $5 \mathrm{meV}$. For a further quantitative analysis, we thus define energy ranges depicted by the red, green, and blue bars in Figs. $1(\mathrm{a})$ and $1(\mathrm{~b})$ corresponding to the $\left(\mathrm{D}^{0}, \mathrm{X}\right),\left(\mathrm{D}^{0}, \mathrm{X}\right)_{\mathrm{s}}$ and FX transitions, respectively. Note that the energy range chosen corresponds in each case to less than the linewidth of the respective emission line at the comparatively high excitation densities $\left(10^{-2} I_{0}\right)$ applied in the experiments.

In order to further confirm the nature of the recombination channels, exemplary excitation-dependent measurements of a single NW are shown in Fig. 1(c). Upon low excitation, a single $\left(\mathrm{D}^{0}, \mathrm{X}\right)$ line at $3.472 \mathrm{eV}$ (as in unstrained $\mathrm{GaN}$ ) with a width of $2 \mathrm{meV}$ is observed. With increasing excitation density, the $\left(\mathrm{D}^{0}, \mathrm{X}\right)$ transition saturates, and the FX line at $3.478 \mathrm{eV}$ becomes dominant. At an intermediate excitation density of $10^{-2} I_{0}$ (blue curve), the same as used for the spectra in Figs. 1(a) and 1(b), the spectrum is actually a superposition of the donor-bound and the free exciton transition with the peak position only slightly shifted, but its linewidth significantly increased with respect to the $\left(D^{0}, X\right)$ line measured at lower excitation. Nevertheless, the linewidth is still sufficiently narrow to distinguish the different transitions participating in recombination. In fact, Fig. 1(d) displays two PL spectra of free-standing NWs, which clearly exhibit several distinct transitions stemming from different recombination channels as indicated. The results shown in Figs. 1(c) and 1(d) confirm that we indeed observe the distinct recombination channels $\left(\mathrm{D}^{0}, \mathrm{X}\right),\left(\mathrm{D}^{0}, \mathrm{X}\right)_{\mathrm{s}}$, and FX to which we have assigned the different excitonic recombination lines in Figs. 1(a) and 1(b). In other words, these spectra do not represent one single transition that is subject to a mechanism causing a continuous energy shift such as strain.

Trichromatic $\mu$-PL area maps of samples A and B are shown in Fig. 2(a). For each pixel in these area maps, the intensity of a given wavelength range is measured and color-coded such that red/orange, green, and blue spots represent spectra dominated by $\left(\mathrm{D}^{0}, \mathrm{X}\right),\left(\mathrm{D}^{0}, \mathrm{X}\right)_{\mathrm{s}}$ and FX transitions, respectively [cf. the horizontal bars in Figs. 1(a) and 1(b)]. Spectra with multiple strong lines result in white or yellow spots in the maps. However, only the spectra of a few NWs exhibit lines resulting from several excitonic transitions. Most spectra are dominated by a single line, either originating from donor-bound (including bulk-like and surface donors) 
(a)
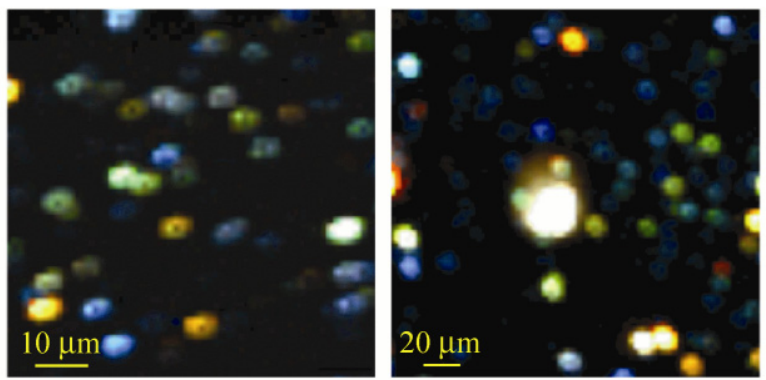

(b)

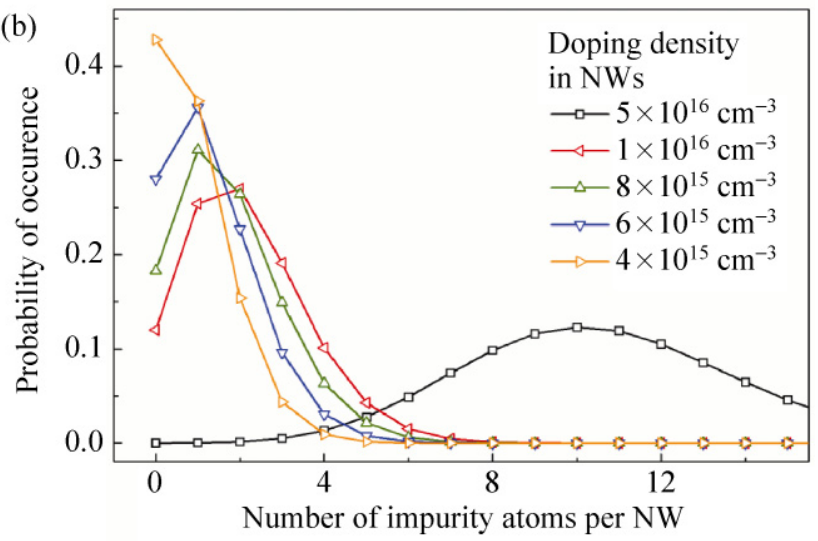

Figure 2 (a) Trichromatic area maps of free-standing NWs for samples A (left) and B (right). The excitation density is $10^{-2} I_{0}$. The large white spot in the center of the map on the right is due to accidentally dispersed NWs and is excluded from further analysis. Saturation and contrast are enhanced to increase the visibility. (b) Number of donors per NW for various doping densities according to Poisson statistics. The NWs have a diameter of $30 \mathrm{~nm}$ and a length of $300 \mathrm{~nm}$

or free exciton emission. In addition to the donor-bound and free exciton emission evident in these spectra, we found that some NWs of sample B also exhibit transitions around $3.45 \mathrm{eV}$ (not shown here), which are characteristic for GaN NWs, but whose origin is still under debate. Transitions related to acceptor-bound excitons at $3.465 \mathrm{eV}$ are not observed at all.

The above observation of a number of NWs emitting only at the FX energy raises a question of particular interest, namely, whether or not the lack of a $\left(\mathrm{D}^{0}, \mathrm{X}\right)$ line is a meaningful signature for the actual absence of a donor in the NW. Conventionally, one would approach this question by comparing the FX diffusion length of at least $200 \mathrm{~nm}$ [12] and the NW dimensions, particularly the length of $300 \mathrm{~nm}$. The exciton thus may or may not experience the short-range potential of the neutral donor and bind to it. However, diffusion is a statistical concept and loses its meaning when dealing with single excitons and donors in the nanoscopic volume of the NW. Considering the sub-wavelength dimensions of the present NWs, the exciton is, at the instant of its creation, a coherent excitation of the entire NW volume with its center-of-mass wavefunction extending throughout the length of the NW [13,14]. In NWs with a diameter $d$ larger than the exciton Bohr radius $a_{\mathrm{B}}$, this effect has been directly observed by the quantization of the center-of-mass motion of the exciton normal to the NW axis [15] (for a theoretical description of the wavefunction and center-of-mass motion of excitons in NWs, see Ref. [16]). A donor located anywhere in the NW represents a perturbation of the crystal symmetry and thus of the coherent extension of the exciton. Due to its attractive potential, the donor spatially localizes the excitonic center-ofmass wavefunction and creates a bound state which manifests its presence by a $\left(\mathrm{D}^{0}, \mathrm{X}\right)$ line in the $\mathrm{PL}$ spectrum.

If these NWs were probed at high excitation density, a single donor would be readily saturated, and FX luminescence would prevail. However, for the excitation density of $10^{-2} I_{0}$ used for recording the map in Fig. 2(a) and considering the measured 1/e decay time of about $130 \mathrm{ps}$, the number of excitons existing at any given time in a NW is actually on the order of one. Consequently, NWs exhibiting only FX transitions most likely do not contain donors at all. Roughly estimated, this is the case for about $25 \%$ of the NWs in the area maps shown in Fig. 2(a).

In a volume as small as that of the present NWs, the number of donors per NW follows a Poisson distribution:

$$
P_{\lambda}(k)=\left(\lambda^{k} / k !\right) \cdot e^{-\lambda}
$$

where $P_{\lambda}(k)$ describes the probability that a NW contains $k$ donor atoms. The probability of an arbitrary atom in the NW being a donor is given by $\lambda$. Figure 2(b) depicts this distribution for different doping densities as determined by Eq. (1). A probability of around $25 \%$ for donor-free NWs with a volume of around $2 \times 10^{-16} \mathrm{~cm}^{3}$ is predicted to be realized for a doping range of $(6-8) \times 10^{15} \mathrm{~cm}^{-3}$, translating into an average 
impurity concentration of one to two atoms per NW. This value is about one order of magnitude lower than the upper limit of $5 \times 10^{16} \mathrm{~cm}^{-3}$ previously obtained from conductance measurements [6]. Figure 2(b) shows that these latter NWs would already contain 10 donors per NW on average. However, recent experimental results from Sanford et al. [17] suggest that the donor concentration in GaN NWs that have not been intentionally doped may be even lower than the value estimated above (down to $5 \times 10^{14} \mathrm{~cm}^{-3}$ ).

Finally, Fig. 1(c) shows that at medium excitation density - as used in the area maps - the peaks may actually be a superposition with contributions from several recombination channels. In order to separate these different contributions, we quantitatively analyze the area maps shown in Fig. 1(a) by fitting each spectrum with four Gaussians, representing the $3.45 \mathrm{eV}$ band and the $\left(\mathrm{D}^{0}, \mathrm{X}\right),\left(\mathrm{D}^{0}, \mathrm{X}\right)_{\mathrm{s}}$, as well as $\mathrm{FX}$ transitions. Figures 3(a) and 3(b) show the distributions of peaks found in the area maps displayed in Fig. 1(a) for samples

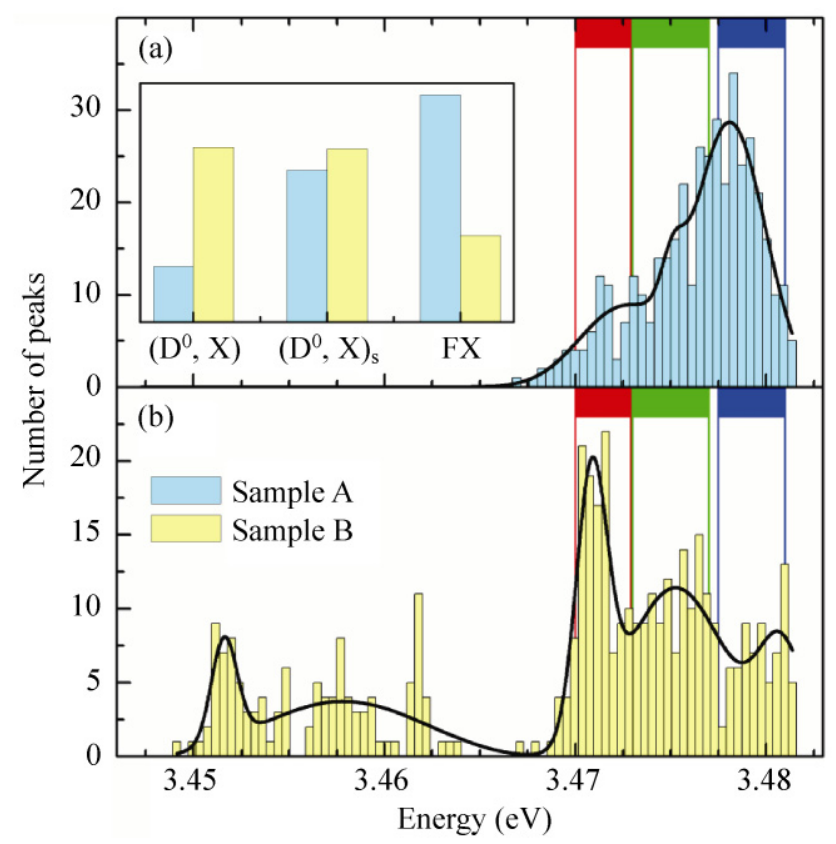

Figure 3 Histograms of peak energies in the area maps for (a) sample A and (b) sample $\mathrm{B}$. The $\left(\mathrm{D}^{0}, \mathrm{X}\right),\left(\mathrm{D}^{0}, \mathrm{X}\right)_{\mathrm{s}}$, and $\mathrm{FX}$ energy ranges are marked by the red, green, and blue bars, respectively. The black lines depict envelopes visualizing the contributions of the individual excitonic transitions. The inset shows the relative occurrence of the three different exciton transitions for the two samples under investigation
A and B, respectively. Note that the histograms show only the number of peaks at a specific wavelength, while their intensity is not taken into account. A green spot in the area maps of Fig. 2(a) may also contain a minor contribution from the $\left(\mathrm{D}^{0}, \mathrm{X}\right)$ transition, which is hidden in the tail of the major, color-determining transition. Both samples exhibit their strongest PL between 3.466 and $3.482 \mathrm{eV}$, with a minor contribution from the $3.45 \mathrm{eV}$ band for sample B. This band actually appears to be a doublet with peaks centered at 3.45 and $3.458 \mathrm{eV}$ as observed by Calleja et al. [18]. We did not, however, find a single NW exhibiting both of these transitions. Apart from this $3.45 \mathrm{eV}$ band, both samples primarily exhibit contributions from $\left(\mathrm{D}^{0}, \mathrm{X}\right)_{\mathrm{s}}$ and FX transitions.

The inset of Fig. 3(a) shows the relative occurrence of the three recombination channels. In addition, Figs. 4(a) and 4(b) show these histograms weighed with the actual intensity, and the inset of Fig. 4(a) depicts the relative intensities of the three recombination

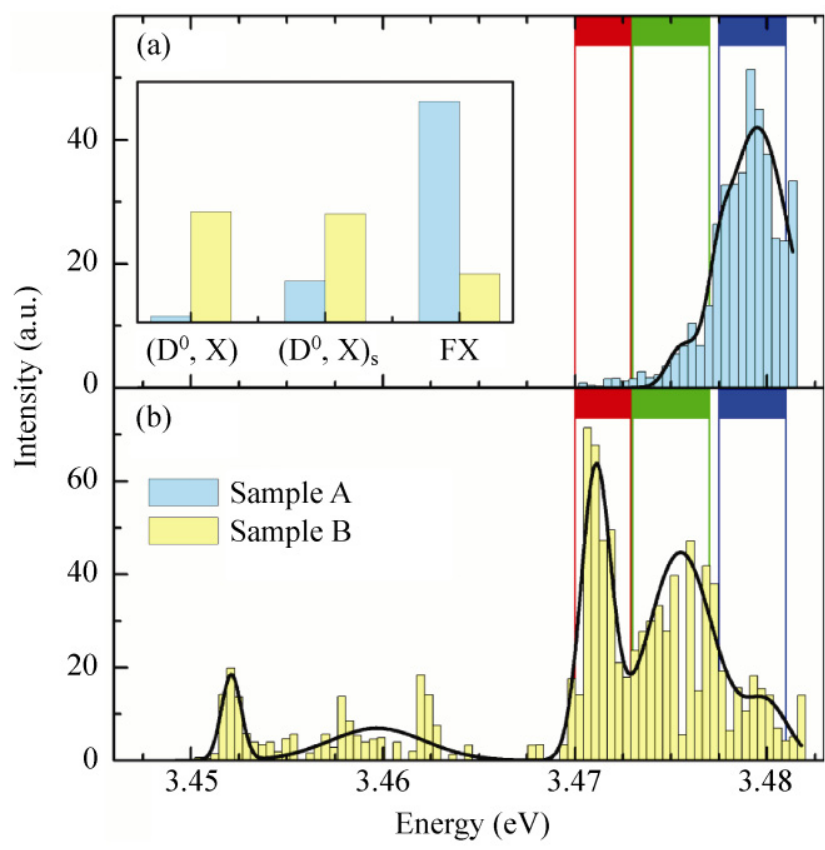

Figure 4 Histograms showing the peak distributions weighed with their intensities. The $\left(D^{0}, X\right),\left(D^{0}, X\right)_{s}$, and $F X$ energy ranges are marked by the red, green, and blue bars, respectively. The envelopes (black lines) visualize the contributions of the individual excitonic transitions. The inset shows the relative intensities of the three different exciton transitions for the two samples under investigation 
channels. The results shown in Figs. 3 and 4 underline that the appearance of the $\left(\mathrm{D}^{0}, \mathrm{X}\right)_{\mathrm{s}}$ transition as described in Ref. [6] is indeed statistically significant, both in terms of number as well as in terms of intensity.

For the investigated NWs, the number of surface donors in sample $\mathrm{A}$ is larger than the number of bulk-like donors, whilst in sample B it is similar to the number of bulk-like donors. Considering their geometry, the high number of $\left(\mathrm{D}^{0}, \mathrm{X}\right)_{\mathrm{s}}$-related peaks is actually not surprising. A significant fraction $\eta$ of donors is located near the surface, i.e. their distance from the surface is less than their Bohr radius $a_{\mathrm{B}}$. With $a$ being the radius of a (cylindrical) NW, $\eta$ is given by

$$
\eta=1-\left(1-a_{\mathrm{B}} / a\right)^{2}
$$

In the present case, $a=15 \mathrm{~nm}$ and $a_{\mathrm{B}}=4.8 \mathrm{~nm}$ [19], yielding $\eta=0.54$ under the assumption of an entirely uniform donor distribution. Surface segregation of impurities $[6,20,21]$ during growth will enhance $\eta$ even further. More important, however, is the large number of FX transitions. For sample $\mathrm{A}$ around $50 \%$ of the observed transitions are related to free excitons, while the corresponding figure for sample B is around $20 \%$. Thus, the histograms demonstrate that the number of NWs without a single donor is very substantial, opening up the possibility of studying excitonpolaritons confined in sub-wavelength NWs.

\section{Conclusions}

We have statistically analyzed the emission of freestanding single GaN NWs. We find that even within a mesoscopic sample area, where maximum homogeneity is assured, the luminescence varies strongly from NW to NW. In the limit of small numbers, statistical fluctuations significantly affect the properties of the NWs, in complete contrast to the case of bulk material. Due to the high crystal quality and the small dimensions of the NWs, the mere presence or absence of a single atom may, for example, determine the electrical transport as well as the optical recombination path. Therefore, the observed fluctuations have serious implications for single NW devices, for which a predictable behavior is desired.

\section{Methods}

GaN NWs were grown by plasma-assisted MBE on $\mathrm{Si}(001)$ and $\mathrm{Si}(111)$ without the use of a catalyst, thus avoiding potential contamination by a foreign element. The base pressure of the MBE system was lower than $10^{-10}$ mbar. During growth, only Ga and N (both having $7 \mathrm{~N}$ purity) were present. Growth occurred at a substrate temperature of $780^{\circ} \mathrm{C}$ and under N-rich conditions $(\mathrm{N} / \mathrm{Ga}=5)$, similar to the conditions described by other groups $[18,22]$. Due to the configuration of the substrate heater in the growth chamber, the wafer experienced a positive temperature gradient toward the edge. As a direct consequence, the nucleation density for GaN was significantly reduced and the nucleation time was considerably prolonged in this region. The time needed for the formation of such a nucleus varied from NW to NW, as thus, also, did their growth time. The resulting NW array had a density of less than $1 \mu \mathrm{m}^{-2}$, which allowed us to optically address individual NWs in a mesoscopic area of $0.04 \mathrm{~mm}^{2}$. Within this area, both the temperature and the atomic fluxes are expected to be essentially perfectly homogeneous. The free-standing NWs in this area were shorter $(\leqslant 300 \mathrm{~nm})$ and smaller in diameter $(\leqslant 30 \mathrm{~nm})$ than those in the center of the wafer which had a length of around $400 \mathrm{~nm}$ and an average diameter of $40 \mathrm{~nm}$. Due to the varying growth times, only NWs that nucleated at the very beginning of the growth process reached a length of $300 \mathrm{~nm}$. Thus, the actual length varied from NW to NW and many NWs were actually just short stubs (50-100 nm). Scanning electron micrographs (shown in Ref. [6]) confirm the complete absence of either coalescence of adjacent NWs or parasitic growth of $\mathrm{GaN}$ between the free-standing NWs. These samples are thus ideally suited for the study of the intrinsic fluctuations of the impurity incorporation in terms of Poisson statistics.

Photoluminescence measurements of the as-grown NWs were performed at a temperature of $10 \mathrm{~K}$. The NW samples were excited by a $\mathrm{HeCd}$ laser with a maximum excitation density of $I_{0}=20 \mathrm{~kW} / \mathrm{cm}^{2}$. The laser spot was focused to a diameter of $3 \mu \mathrm{m}$ by an ultraviolet microscope objective with a numerical aperture of 0.32 . The same objective was used to collect 
and focus the PL signal onto the entrance slit of an $80-\mathrm{cm}$ spectrometer. One of two gratings with 600 or 2400 lines/mm was applied to disperse the PL signal spectrally, after which it was detected by a liquid nitrogen cooled charge-coupled device array. In order to perform these measurements in a manageable time span, the integration time for each spectrum was limited to $2 \mathrm{~s}$ at an excitation density of $10^{-2} I_{0}$.

The quantitative analysis of the PL area maps consisted of several steps: First, spectra recorded in the area between NWs (black spaces in the maps) and also spectra governed by noise were discarded by applying an intensity threshold. Note that most of the latter stem from NW stubs. In principle, these NWs could have been included in the analysis if the noise level was reduced by using a longer integration time. This concerns about $50 \%$ of the NWs from sample A and about $80 \%$ from the NWs of sample B. The remainning spectra were fitted with a set of four Gaussians, representing the $3.45 \mathrm{eV}$ band, $\left(\mathrm{D}^{0}, \mathrm{X}\right),\left(\mathrm{D}^{0}, \mathrm{X}\right)_{\mathrm{s}}$, and $\mathrm{FX}$ transitions. No restrictions were applied to the fitting parameters. For each set, the peak with the maximum ratio $r_{\max }$ of peak height to linewidth was determined. In order to reduce the influence of spurious signals due to noise, the remaining peaks $(25 \%$ for sample A and $55 \%$ for sample B) were considered further only if their ratio $r_{i} \geqslant 0.1 r_{\max }$. Peaks with $r_{i}<0.1 r_{\max }$ were omitted.

\section{Acknowledgements}

We greatly appreciate the fruitful discussions with Vladimir Kaganer and are indebted to Uwe Jahn for cathodoluminescence measurements as well as for a critical reading of the manuscript. This work was partially supported by the EU Marie Curie RTN contract MRTN-CT-2004-005583 (PARSEM) and by the IST project NODE 015783.

Open Access: This article is distributed under the terms of the Creative Commons Attribution Noncommercial License which permits any noncommercial use, distribution, and reproduction in any medium, provided the original author(s) and source are credited.

\section{References}

[1] International Technology Roadmap for Semiconductors. http://www.itrs.net (accessed Aug 3, 2010).

[2] Stach, E. A.; Pauzauskie, P. J.; Kuykendall, T.; Goldberger, J.; He, R.; Yang, P. Watching GaN nanowires grow. Nano Lett. 2003, 3, 867-869.

[3] Lieber, C. M.; Wang, Z. L. Functional nanowires. MRS Bull. 2007, 32, 99-104 and references therein.

[4] Czaban, J. A.; Thompson, D. A.; LaPierre, R. R. GaAs core-shell nanowires for photovoltaic applications. Nano Lett. 2009, 9, 148-154.

[5] Chèze, C.; Geelhaar, L.; Trampert, A.; Riechert, H. In situ investigation of self-induced $\mathrm{GaN}$ nanowire nucleation on Si. Appl. Phys. Lett. 2010, 97, 043101.

[6] Brandt, O.; Pfüller, C.; Chèze, C.; Geelhaar, L.; Riechert, H. Sub-meV linewidth of excitonic luminescence in single GaN nanowires: Direct evidence for surface excitons. Phys. Rev. $B$ 2010, 81, 045302 .

[7] Love, A. E. H. A Treatise on the Mathematical Theory of Elasticity; Dover: New York, 1944.

[8] Wysmolek, A.; Korona, K. P.; Stępniewski, R.; Baranowski, J. M.; Błoniarz, J.; Potemski, M.; Jones, R. L.; Look, D. C.; Kuhl, J.; Park, S. S.; Lee, S. K. Recombination of excitons bound to oxygen and silicon donors in freestanding GaN. Phys. Rev. B 2002, 66, 245317.

[9] Muljarov, E. A.; Zhukov, E. A.; Dneprovskii, V. S.; Masumoto, $Y$. Dielectrically enhanced excitons in semiconductor-insulator quantum wires: Theory and experiment. Phys. Rev. B 2000, 62, 7420-7432.

[10] Levine, J. D. Nodal hydrogenic wave functions of donors on semiconductor surfaces. Phys. Rev. 1965, 140, A586-A589.

[11] Diarra, M.; Niquet, Y. -M.; Delerue, C.; Allan, G. Ionization energy of donor and acceptor impurities in semiconductor nanowires: Importance of dielectric confinement. Phys. Rev. B 2007, 75, 045301.

[12] Ino, N.; Yamamoto, N. Low temperature diffusion length of excitons in gallium nitride measured by cathodoluminescence technique. Appl. Phys. Lett. 2008, 93, 232103.

[13] Hanamura, E. Rapid radiative decay and enhanced optical nonlinearity of excitons in a quantum well. Phys. Rev. B 1988, 38, 1228-1234.

[14] Dubin, F.; Melet, R.; Barisien, T.; Grousson, R.; Legrand, L.; Schott, M.; Voliotis, V. Macroscopic coherence of a single exciton state in an organic quantum wire. Nat. Phys. 2006, 2, 32-35. 
[15] Lage, H.; Heitmann, D.; Cingolani, R.; Grambow, P.; Ploog, $\mathrm{K}$. Center-of-mass quantization of excitons in GaAs quantumwell wires. Phys. Rev. B 1991, 44, 6550-6553.

[16] Siarkos, A. Exciton center-of-mass motion in quantum wells and quantum wires. PhD dissertation, Humboldt-Universität zu Berlin, Berlin 2000.

[17] Sanford, N. A.; Blanchard, P. T.; Bertness, K. A.; Mansfield, L.; Schlager, J. B.; Sanders, A. W.; Roshko, A.; Burton, B. B.; George, S. M. Steady-state and transient photoconductivity in $c$-axis $\mathrm{GaN}$ nanowires grown by nitrogen-plasma-assisted molecular beam epitaxy. J. Appl. Phys. 2010, 107, 034318.

[18] Calleja, E.; Sánchez-García, M. A.; Sánchez, F. J.; Calle, F.; Naranjo, F. B.; Muñoz, E.; Jahn, U.; Ploog, K. H. Luminescence properties and defects in $\mathrm{GaN}$ nanocolumns grown by molecular beam epitaxy. Phys. Rev. B 2000, 62,
16826-16834.

[19] Pedrós, J.; Takagaki, Y.; Ive, T.; Ramsteiner, M.; Brandt, O.; Jahn, U.; Ploog, K. H.; Calle, F. Exciton impact-ionization dynamics modulated by surface acoustic waves in GaN. Phys. Rev. B 2007, 75, 115305.

[20] Fernández-Serra, M. V.; Adessi, Ch.; Blase, X. Surface segregation and backscattering in doped silicon nanowires. Phys. Rev. Lett. 2006, 96, 166805.

[21] Dalpian, G. M.; Chelikowsky, J. R. Self-purification in semiconductor nanocrystals. Phys. Rev. Lett. 2006, 96, 226802.

[22] Meijers, R.; Richter, T.; Calarco, R.; Stoica, T.; Bochem, H. P.; Marso, M.; Lüth, H. GaN-nanowhiskers: MBE-growth conditions and optical properties. J. Cryst. Growth 2006, 289, 381-386. 